\title{
Leitlinien-Initiative der DEGUM
}

Vor einigen Jahren hat die DEGUM die Initiative ergriffen die Leitlinienarbeit qualitativ und methodisch zu verbessern. So hat der Vorstand bereits Ende 2016 Professor Constantin von Kaisenberg, Bereichsleiter Geburtshilfe und Pränatalmedizin des Perinatalzentrum der Medizinischen Hochschule Hannover, zum Leitlinienbeauftragten der gesamten DEGUM ernannt. Ziel ist es zukünftig Leitlinien der DEGUM als AWMFLeitlinien zu erstellen. Dazu erwarb Professor von Kaisenberg das Zertifikat „AWMFzertifizierter Leitlinien-Berater“.

Im Juni 2018 haben die DEGUM und die Deutsche Gesellschaft für Gynäkologie und Geburtshilfe (DGGG) eine Kooperationsvereinbarung getroffen. „Dies stellte eine Konzentration zweier Fachgesellschaften dar, weil sich dadurch eine sehr wichtige politische Synergie ergab“, betont Professor von Kaisenberg. Die beiden Fachgesellschaften einigten sich darauf, dass sie bei allen AWMF-Leitlinien, die Ultraschall in der Pränatalmedizin und Geburtshilfe betreffen, künftig kooperieren wollen. „Dies bedeutet, dass beide Gesellschaften als anmeldende Fachgesellschaften eingetragen werden und beide Gesellschaften eine Person benennen, die als Koordinator eingetragen wird“, so Professor von Kaisenberg. Entsprechend bestehe eine paritätische Federführung.

Im gleichen Jahr hat der Vorstand eine Standardvorgehensweise zur LeitlinienErstellung verabschiedet. Darin sind die Antragstellung für eine Leitlinien-Anmeldung bei der AWMF, die Finanzierung und der Erstellungsprozess sowie die Berichtspflicht detailliert beschrieben. Weiterhin wird in der Standardvorgehensweise unterschieden zwischen den verschiedenen Formen der Beteiligungen an einer Leitlinie, die federführend durch die DEGUM erfolgen kann, paritätisch mit einer anderen Fachgesellschaft oder teilnehmend bei anderen Fachgesellschaften. Dies dient der Unterstützung bei der Erstellung und bei der Publikation von Leitlinien.

Anfang 2020 nun hat sich der Vorstand erneut mit den Aufgaben des LeitlinienBeauftragten beschäftigt und eine Strategie für die Leitlinienarbeit der DEGUM verabschiedet. Darin heißt es: „Leitlinien beschreiben einen Korridor für die standardisierte Diagnostik und Therapie von Patienten im deutschsprachigen Raum. Hierfür werden die qualitativ bestmöglichen klinischen und wissenschaftlichen Studien zugrunde gelegt und mithilfe der modernsten Methoden bewertet. Dies stellt die Grundlage unseres ärztlichen Handelns dar. Die Erstellung qualitativ hochwertiger Leitlinien ist daher eine zentrale Tätigkeit einer wissenschaftlichen Fachgesellschaft.“ Die DEGUM unterhält zudem enge Kontakte zum Royal College of Obstetricians and Gynecologists (RCOG), der National Guideline Alliance (NGA) und dem National Institute for Health and Care Excellence (NICE), was einen Wissens- und Methodentransfer für die Leitlinienarbeit ermöglicht.

Außerdem wurde ein Leitlinien-Register angelegt, das der Administration der Leitlinien, der Planung neuer Leitlinien und der strategischen, politischen, vor allem aber jedoch wissenschaftlichen Ausrichtung der DEGUM dient. Es erleichtert außerdem die Zusammenarbeit an einer Leitlinie durch einen online-Zugang.

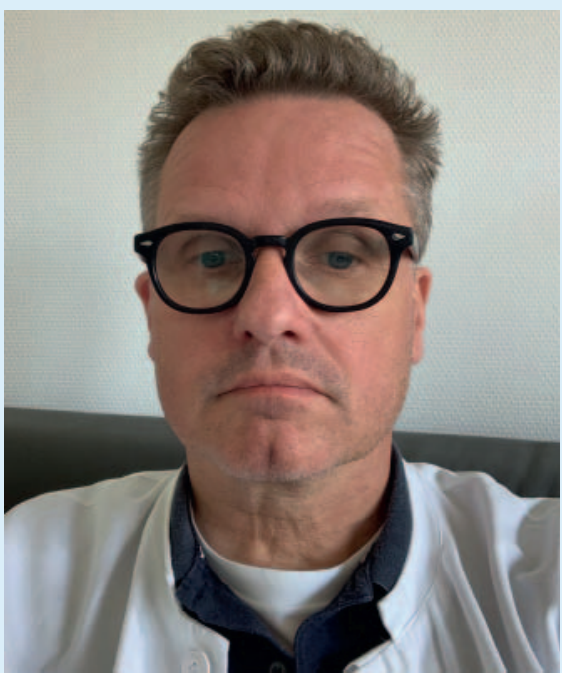

Leitlinienbeauftragter Prof. Dr. Constantin von Kaisenberg

„Weiterhin wurde eine Leitlinien-Kommission eingerichtet, deren Ziel die Kommunikation mit den Vorsitzenden der Sektionen und Sprechern der Arbeitskreise ist, um wissenschaftlich interessante und wichtige Themen zu identifizieren und zur Leitlinien-Anmeldung zu führen“, so Professor von Kaisenberg. Die Kommission wird alle Sektionen und Arbeitskreise der DEGUM bei ihren Leitlinien-Vorhaben administrativ und methodisch unterstützen.

Es wird zudem eine enge Kooperation mit anderen Fachgesellschaften angestrebt. Dies macht die DEGUM besser als lebendige und wissenschaftlich aktive Fachgesellschaft wahrnehmbar. So kommt die DEGUM ihrem Ziel näher, qualitativ und methodisch hochwertige und aktuelle Leitlinien zu erstellen. 
Die erste bei der AWMF von der DEGUM (paritätisch mit der DGGG) angemeldete LL:

- 085-002 S2e Ersttrimester Diagnostik und Therapie @ 11-13 ${ }^{+6}$ Schwangerschaftswochen

Die erste bei der AMWF von der DEGUM federführend angemeldete LL:

- 085-003 S2e Fraktursonographie

Die erste bei der AWMF paritätisch mit DEGUM und DGGG fertig gestellte LL ist die:

- 015-087 S2e LL Überwachung und Betreuung von Zwillingsschwangerschaften

Bei weiteren AWMF LL ist die DEGUM interdisziplinär aktiv beteiligt, u. a.:

Pränatalmedizin \& Geburtshilfe:

- 015-084 S3 Sectio caesarea

- 015-083 S3 Vaginale Geburt am Termin, LL Anmeldung

- 015-089 S3 Fetale Überwachung (Indikation und Methodik zur fetalen Zustandsdiagnostik in der Schwangerschaft), LL Anmeldung

- 022-028 S2k Klassifikation und Diagnostik der Mikrozephalie

- 166-003 S2k Nierenzysten und zystische Nierenerkrankungen bei Kindern

\section{Hämatologie \& Onkologie:}

- 018-029OL S3 Diagnostik, Therapie und Nachsorge des Hodgkin Lymphoms bei erwachsenen Patienten

- 018-032OL S3 Diagnostik, Therapie und Nachsorge für Patienten mit einer chronisch lymphatischen Leukämie

- 018-033OL S3 Diagnostik, Therapie und Nachsorge für Patienten mit einem follikulären Lymphom

- 021-023OL S3 Diagnostik und Therapie der Plattenepithelkarzinome und Adenokarzinome des Ösophagus

- 031-056OL S3 Schilddrüsenkarzinom, LL Anmeldung

- 032-009 OL S3 Magenkarzinom - Diagnostik und Therapie der Adenokarzinome des Magens und ösophagogastralen Übergangs

- 032-010 OL S3 Exokrines Pankreaskarzinom

- 032-024 OL S3 Diagnostik, Therapie und Nachsorge des Melanoms

- 032-045 OL S3 Früherkennung, Diagnostik, Therapie und Nachsorge des Mammakarzinoms

- 032-053 OL S3 Hepatozelluläres Karzinom, Diagnostik und Therapie

\section{Viszeralmedizin:}

- 021-003 S3 Chronische Pankreatitis

- 021-008 S3 Diagnostik und Therapie von Gallensteinen

- 021-017 S2k Komplikationen der Leberzirrhose

- 021-020 S2k Divertikelkrankheit/Divertikulitis

- 021-022 S2k Gastrointestinale Endoskopie, Qualitätsanforderungen

- 021-025 S2k Nicht-alkoholische Fettlebererkrankungen

- 021-027 S2k Autoimmune Lebererkrankungen (AILE)

\section{Gefäßmedizin:}

- 004-014 S3 Screening, Diagnostik, Therapie und Nachsorge des Bauchaortenaneurysmas

- 064-003 S3 Periphere arterielle Verschlusskrankheit (PAVK), Diagnostik, Therapie und Nachsorge

weitere:

- 007-091 S3 Antiresorptiva-assoziierte Kiefernekrose (AR-ONJ)

- 021-023OL, S3, Diagnostik und Therapie der Plattenepithelkarzinome und Adenokarzinome des Ösophagus

Weitere Informationen zu den Leitlinienaktivitäten der DEGUM finden Sie auf der Homepage unter www.degum.de/aktivitaeten/leitlinien. 